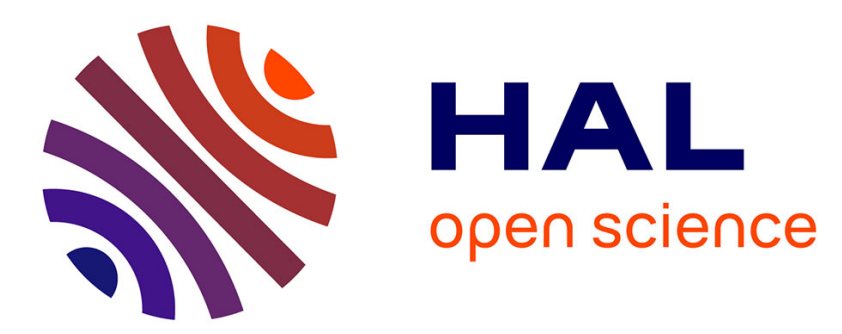

\title{
Quasi-one-dimensional superconductors in strong magnetic field
}

\author{
N. Dupuis, G. Montambaux, C.A.R. Sá de Melo
}

\section{To cite this version:}

N. Dupuis, G. Montambaux, C.A.R. Sá de Melo. Quasi-one-dimensional superconductors in strong magnetic field. Journal de Physique IV Proceedings, 1993, 03 (C2), pp.C2-311-C2-314. 10.1051/jp4:1993263 . jpa-00251346

\section{HAL Id: jpa-00251346 https://hal.science/jpa-00251346}

Submitted on 1 Jan 1993

HAL is a multi-disciplinary open access archive for the deposit and dissemination of scientific research documents, whether they are published or not. The documents may come from teaching and research institutions in France or abroad, or from public or private research centers.
L'archive ouverte pluridisciplinaire HAL, est destinée au dépôt et à la diffusion de documents scientifiques de niveau recherche, publiés ou non, émanant des établissements d'enseignement et de recherche français ou étrangers, des laboratoires publics ou privés. 


\title{
Quasi-one-dimensional superconductors in strong magnetic field
}

\author{
N. DUPUIS, G. MONTAMBAUX and C.A.R. SÁ DE MELO*
}

Laboratoire de Physique des Solides, Université Paris-Sud, 91405 Orsay, France

* Science and Technology Center for Superconductivity, Argonne National Laboratory, Materials Science Division, Argonne IL 60439, U.S.A.

We determine the phase diagram of a quasi-one-dimensional conductor (weakly coupled chains system with an open Fermi surface) in a magnetic field. The usual Ginzburg-Landau regime is followed, when the field is increased, by a cascade of superconducting phases separated by first order transitions, which ends with a strong reentrance of the superconducting phase. These new phases show a novel kind of symmetry of laminar type. The Zeeman splitting does not completely suppress the reentrance in very strong field, the ground state being in this case a Larkin-Ovchinnikov-Fulde-Ferrell state.

\section{Introduction}

The usual theory of type II superconductivity treats the magnetic field in the semiclassical phase integral approximation which is correct only if $\omega_{c} \ll T, 1 / \tau\left(\omega_{c}\right.$ is the caracteristic energy and $\tau$ is the elastic scattering time). In sufficiently clean materials, it will break down at low temperature. It has recently been pointed out that when the quantum effects of the field (i.e. the Landau level quantization in isotropic superconductors) are considered, the superconducting phase may survive at high magnetic field contrary to the predictions of the Ginzburg-Landau theory [1].

We have studied the quantum effects of the magnetic field in a quasi-one-dimensional superconductor with an open Fermi surface described by the dispersion law:

$E(\mathbf{k})=v\left|k_{x}\right|+t_{y} \cos \left(k_{y} b\right)+t_{z} \cos \left(k_{z} c\right)$,

where $v$ is the Fermi velocity for the motion along the chains, and $t_{y}, t_{z}$ are the coupling between chains. For a magnetic field along the $y$ direction, the semiclassical electronic orbits are open and of the form $z=c\left(t_{z} / \omega_{c}\right) \cos (G x)$ where $G=-e H c$ and $\omega_{c}=G v$. Consequently, there is no Landau level quantization but the field localizes the electrons in the $z$ direction. In very strong field $\left(\omega_{c} \gg t_{z}\right)$, the amplitude of the motion becomes smaller than the distance $c$ between chains, showing that the motion is two-dimensional : the magnetic field being parallel to the planes of the electronic motion, the time reversal invariance is restored so that the orbital frustration of the order parameter disappears. Because of this magnetic field induced two-dimensionalization, the superconducting phase is always 
stable at low temperature and exibits a strong reentrance in high magnetic field for equal spin triplet pairing as was first shown by Lebed' [2].

\section{Transition line $T_{c}(H)$}

The zero field critical temperature is assumed to be smaller than the coupling between chains $\left(T_{c} \ll t_{y}, t_{z}\right)$ : this condition ensures that the superconductivity is always of 3D type (and not of Josephson type) at all temperature. When quantum effects of the field are not considered, such a superconductor is well described by the anisotropic Ginzburg-Landau theory: the superconducting state is an anisotropic vortex lattice, the normal state being restored at a field $H_{c 2}(T)=\phi_{0} / 2 \pi \xi_{x}(T) \xi_{z}(T)$, where $\phi_{0}$ is the flux quantum and $\xi_{x}, \xi_{z}$ are the coherence lengths.

(a)

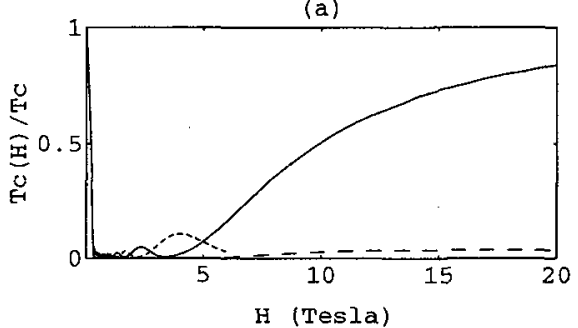

(b)

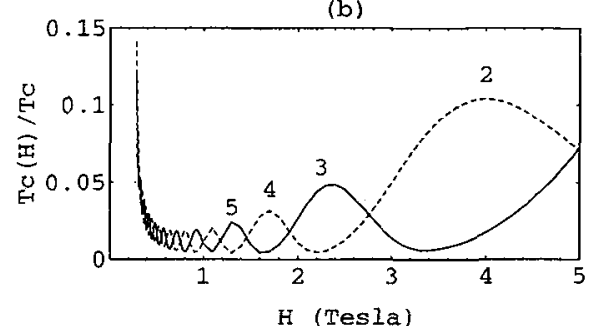

H (Tesla)

Figure 1: (a) Critical temperature $T_{c}(H) / T_{c}$ versus magnetic field ( $t_{z}=20 \mathrm{~K}$ and $t_{z} / T_{c} \sim 13$ ). Triplet pairing: solid and dashed lines; singlet pairing: long-dashed line. (b) The low temperature region for the triplet case. Each phase is labeled by an integer $N$ (see text).

The critical line $T_{c}(H)$ has been obtained in a mean field (BCS) theory where the quantum effects of the magnetic field $\mathbf{H}(0, H, 0)$ are fully taken into account (Fig.1) [3]. In the weak field regime $\left(\omega_{c} \ll T\right)$, the critical temperature decreases when the field is increased in agreement with the Ginzburg-Landau theory. In the quantum regime $\left(\omega_{c} \gg T\right)$, there are two lines of instability (solid line and dashed line on Fig.1) which correspond to two different structures of the order parameter. Thus, the field induces a cascade of superconducting phases separated by first order transitions. The resulting phase diagram is somehow reminiscent of the field-induced spin-density-wave phases [4]. In the very strong field regime $\left(\omega_{c} \gg t_{z}\right)$, the system shows a quasi-2D behavior as a consequence of the magnetic field induced two-dimensionalization : the superconducting long-range order is ensured by a 
Josephson coupling between the chains in the $z$ direction. When the Zeeman splitting effect is taken into account, the critical temperature is strongly reduced for singlet pairing. However, this effect can be partially compensated by an additional spacial modulation of the order parameter along the $x$ direction, the ground state being in this case a Larkin-Ovchinnikov-Fulde-Ferrell (LOFF) state [5]. As shown on Fig.1 (long-dashed line), the reentrance of the superconducting phase is not completely suppressed.

\section{Ordered phase}

In order to obtain the order parameter in the ordered phase, we have followed the approach originally proposed by Abrikosov for the study of type II superconductivity: the order parameter at $T<T_{c}(H)$ is written as a linear combination of the solutions of the linearized gap equation at $T_{c}(H)$. This linear combination is chosen so that the weak field $\left(\omega_{c} \ll T\right)$ solution is an Abrikosov vortex lattice and the very strong field $\left(\omega_{c} \gg t_{z}\right)$ solution is a Josephson vortex lattice. We take into account the discretness along the $z$ direction by requiring the order parameter to have periodicity $a_{z}=N c(N$ integer). In the weak field regime $\left(\omega_{c} \ll T\right)$, the superconducting state is an Abrikosov vortex lattice whose unit cell $\left(a_{x}, a_{z}\right)$ is determined by the coherence lengths $\xi_{x}(T)$ and $\xi_{z}(T)$ (Fig.2a) (for simplicity, we consider a square vortex lattice). In the quantum regime $\left(\omega_{c} \gg T\right)$, the transverse periodicity $a_{z}$ is not determined by the coherence length $\xi_{z}(T)$ anymore but by the transverse magnetic length which corresponds to the amplitude in the $z$ direction of the semiclassical motion. In these quantum phases, the order parameter shows a symmetry of laminar type (Fig.2b). Fach phase corresponds to a given periodicity $N$ of the order parameter. The cascade of phase transitions is then due to commensurability effects between the crystalline lattice spacing $c$ and the periocity $a_{z}$ of the order parameter. In very strong field, the electrons become localized in the planes $z=m c$. Therefore the last phase corresponds to $N=1$ (this choice $N=1$ is possible because of the square symmetry of the order parameter). This phase corresponds to a square lattice of Josephson vortices.
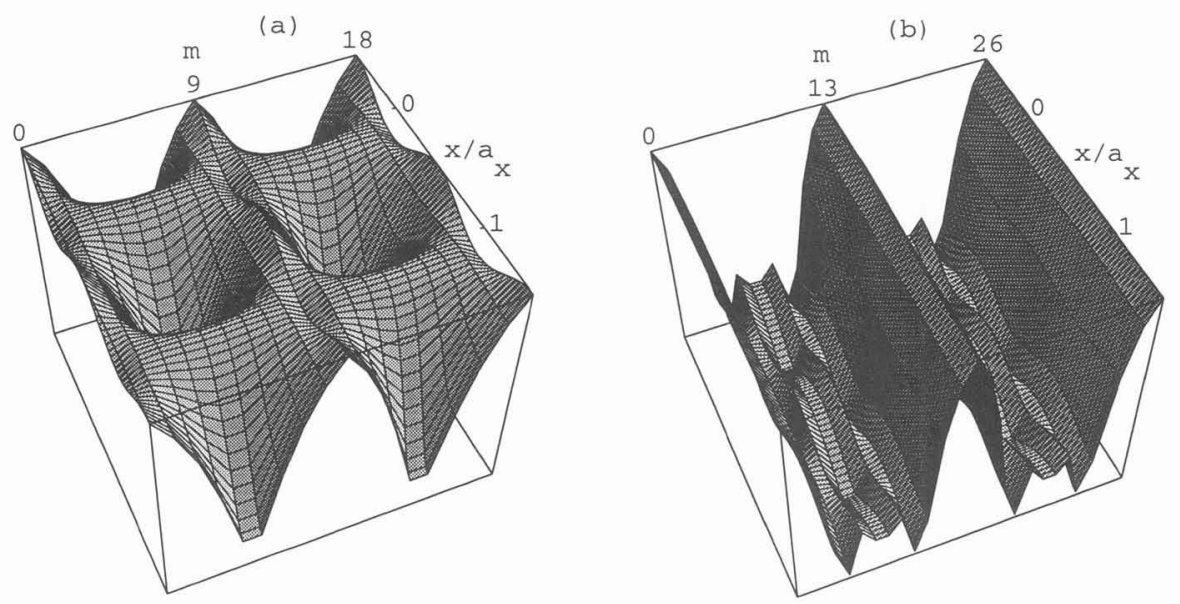

Figure 2: Order parameter $|\Delta(x, z=m c)|$. (a) weak field regime $\omega_{c} \ll T$. (b) quantum regime $\omega_{c} \gg T$. 


\section{Possible experimental consequences}

In Bechgaard salts like (TMTSF) ${ }_{2} \mathrm{ClO}_{4}$ and (TMTSF) ${ }_{2} \mathrm{PF}_{6}$, the phase diagram proposed in this paper could be observed. The intermediate phases involve a low temperature range which can be reached experimentally only in the case of triplet superconductivity $\left(T_{c}(H) \sim 10 \mathrm{mK}\right)$. The reentrance in high field should be visible even in the case of singlet superconductivity $\left(T_{c}(H) \sim 50 \mathrm{mK}\right)$.

Similar quantum effects are also expected to be observed in quasi-two-dimensional superconductors in a magnetic field parallel to the superconducting planes [6]. Although the situation is more complicated due to the presence of open and closed orbits at the Fermi surface, it can be shown from semiclassical arguments that the field induces a $3 \mathrm{D} / 2 \mathrm{D}$ crossover. Consequently, the phase diagram proposed in this paper (Fig.1) should also hold for quasi-2D superconductors. It should be noted that the effect of Zeeman splitting is much more prononced than in the quasi-one-dimensional case. The reason is that the LOFF state compensates the Pauli pair breaking effect very weakly for a $2 \mathrm{D}$ isotropic Fermi surface. Therefore, the possibility to observe the reentrance of the superconducting phase is very weak unless the Pauli limited field is very large. Nevertheless, quantum effects appear also at low temperature for fields near $H_{c 2}(0)$ (calculated in the Ginzburg-Landau theory) where the Pauli pair breaking effect is negligeable for most of the type II superconductors. The corresponding temperature is of order of $T^{*} \sim T_{c}^{2} / t_{z}$ where $t_{z}$ is the coupling between planes. For a sufficiently weak coupling $t_{z}, T^{*}$ will be high enough to be reached experimentally without any difficulty. Therefore, the ratio $T_{c} / t_{z}$ should not be too small in order to see quantum effects at reasonable temperature. Among possible candidates for the observation of superconductivity in strong magnetic field, we can think of quasi-2D organic superconductors or artificial superlattices of superconductors.

\section{REFERENCES}

1. For a review, see M. Rasolt and Z. Tešanović, Rev. Mod. Phys. 64, 709 (1992).

2. A.G. Lebed', JETP Lett. 44, 114 (1986); L.I. Burlachkov, L.P. Gor'kov and A.G Lebed', EuroPhys. Lett. 4, 941 (1987).

3. N. Dupuis, G. Montambaux and C.A.R. Sá de Melo, Phys. Rev. Lett. 70, 2613 (1993).

4. For a review, see G. Montambaux, Phys. Scr. T35, 188 (1991).

5. P. Fulde and R.A. Ferrell, Phys. Rev. 135, A550 (1964); A.I. Larkin and Yu.N. Ovchinnikov, Sov. Phys. JETP 20, 762 (1965).

6. N. Dupuis, $\mathrm{PhD}$ thesis, Université Paris-Sud, unpublished (1993). 